\title{
ROBUST IDENTIFICATION OF PROCESS MODELS FROM PLANT DATA
}

\author{
Graham C. Goodwin*,1, Juan C. Agüero*, James S. Welsh*, \\ Gregory J. Adams*, Juan I. Yuz ${ }^{* * 2}$ and Cristian R. Rojas* \\ ${ }^{*}$ Centre for Complex Dynamic Systems and Control (CDSC). \\ The School of Electrical Engineering and Computer Science, \\ The University of Newcastle, Australia. \\ ${ }^{* *}$ Electronics Department, Universidad Técnica Federico Santa \\ María, Valparaíso, Chile.
}

\begin{abstract}
A precursor to any advanced control solution is the step of obtaining an accurate model of the process. Suitable models can be obtained from phenomenological reasoning, analysis of plant data or a combination of both. Here, we will focus on the problem of estimating (or calibrating) models from plant data. A key goal is to achieve robust identification. By robust we mean that small errors in the hypotheses should lead to small errors in the estimated models. We argue that, in some circumstances, it is essential that special precautions be taken to ensure that robustness is preserved. We present several practical case studies to illustrate the results. Copyright @2007 IFAC.
\end{abstract}

Keywords: Closed loop identification, robust identification.

\section{INTRODUCTION}

The word robust has, in recent years become inextricably linked to advanced control. This has been an important step with significant practical consequences. However, by way of contrast, little has been explicitly written about robust identification, although the idea is implicit in much of the previous literature (Ljung, 1999). Nonetheless, robustness issues play a central role in successful identification experiments.

With the above as background the current paper is concerned with robust identification of process models from plant data.

One way of thinking about robustness is that small changes in the working hypotheses should lead to small changes in the end result. This may seem rather obvious, but surprisingly, robustness issues are sometimes overlooked. For example, we know of an in-

1 Graham.Goodwin@newcastle.edu.au

2 Work supported by grant FONDECYT 3060013, Chile. dustrial case study where a $40^{\text {th }}$ order non-minimum phase model was fitted to a particular plant. It subsequently turned out that a more robust procedure showed that a much better description was provided by a simple integrator plus time delay.

Here, we will suggest various strategies for robustifying solutions. Often, this amounts to detuning the algorithm such that it is not overly sensitive to the hypotheses. Inevitably a price is paid in terms of nominal performance. However, this is often a relatively small price to pay for robustness.

The specific problems that we will study include

- selecting model class,

- sampling,

- experiment design, and

- closed loop identification.

In each case we will show that there exists a potential robustness problem. We will then suggest simple methods which can be used to improve robustness. 


\section{MOTIVATING PRACTICAL CASE STUDY}

Before addressing the various technical issues we will briefly review a real world system identification study which shows some of the main difficulties encountered in practical identification studies. This system will highlight many of the issues that we will address in more detail in the sequel. The specific system is a "nutating grinding mill".

The identification experiments were tested on a full nonlinear simulation model since the real system was under development.

Grinding mills used in mineral processing (NapierMunn et al., 1996) produce material of an appropriate size to either (a) enable downstream processes to liberate the material of interest, or (b) produce finished products. Here we focus on a new mill design called a Hicom nutating grinding mill (Hoyer and Boyes, 1990).

The available control system variables (see Figure 1) are:

- Mill feed rate.

- Mill speed.

- Classifier rotor speed.

- System air.

- Secondary air rate to classifier.

- Dilution air rate to mill discharge.

- Grinding media (periodic, manual addition).

The process variables are

- Product specification (desired percentage of material passing a set product size).

- Circulating load ratio (flow rate of product divided by flow rate of recycled material).

- Grinding efficiency (Mill power divided by product rate).



Fig. 1. Hicom Mill configuration, showing measured inputs and outputs.

The final control system needs to respect many constraints. Hence, a constrained Model Predictive Control law will be used. Also, the system should not be operated in open loop. Hence the system identification will be carried out using closed loop data and a constrained control law (see later sections where we discuss closed loop identification and virtual closed loops).
The final control system needs to incorporate selflearning capabilities, as there is limited time for commissioning of the control system during installation, and the mill operators are assumed to have limited technical ability. Therefore it is necessary to utilize closed-loop identification schemes (i.e. those that can run with a system under feedback control) to determine a useful model of the mill circuit. Small excitation signals (sine waves) are injected into the setpoints. Note that the system is very nonlinear and a series of local linear models are required at different operating points. Closed loop operation is again helpful in defining and maintaining these points.

The overall time constant of the grinding operation depends on the residence time of the mill, which is long for low rates of material breakage. Thus manual determination of the residence time (via the dynamic response of the circulating load ratio) is needed during commissioning.

Once this is determined, the closed loop identification test duration is chosen to be a multiple of this. The lowest frequency sinewave is chosen to have a frequency less than the circuit bandwidth. For example, the maximum residence time for the simulated model has been determined to be around 60 seconds, giving a test duration of 1200 seconds (20 minutes).

We propose to use direct identification (see later section). We first obtain the empirical transfer function estimate using frequency domain analysis. Next, we fit parameterized models to each component of the frequency response. Say we have $p$ process variables $(Y), m$ manipulated variables $(U)$ and $s$ sets of data at a given frequency, then the input-output model can be with as

$$
\begin{aligned}
& {\left[\begin{array}{cccc}
Y_{11} & Y_{12} & \cdots & Y_{1 s} \\
Y_{11} & Y_{12} & \cdots & Y_{1 s} \\
\vdots & \vdots & \vdots & \\
Y_{p 1} & Y_{p 2} & \cdots & Y_{p s}
\end{array}\right]=} \\
& {\left[\begin{array}{cccc}
G_{11} & G_{12} & \cdots & G_{1 m} \\
G_{11} & G_{12} & \cdots & G_{1 m} \\
\vdots & \vdots & \vdots & \\
G_{p 1} & G_{p 2} & \cdots & G_{p m}
\end{array}\right]\left[\begin{array}{cccc}
U_{11} & U_{12} & \cdots & U_{1 s} \\
U_{11} & U_{12} & \cdots & U_{1 s} \\
\vdots & \vdots & \vdots & \\
U_{m 1} & U_{m 2} & \cdots & U_{m s}
\end{array}\right]}
\end{aligned}
$$

Note that, even though the reference signals may be excited separately, the action of the closed loop means that in each test all inputs will be excited.

When $s=m$, the components of the $p \times m$ frequency response can be obtained by inversion and when $s>$ $m$, then one can use some form of generalized inverse.

An initial closed loop control design was based on this model and was found to perform satisfactorily for the non-linear simulation model.

Motivated by the above example we next turn to the technical issues. 


\section{SELECTING A MODEL CLASS}

\subsection{The role of physics}

The first issue to be addressed in a system identification exercise is the specification of the class of models to be fitted. Here the physics of the problem plays a central role in achieving robustness. This does not mean that one has to develop a large scale, distributed parameter model. Indeed, quite to the contrary, a large scale model may contain far too many free parameters to be calibrated. The key thing is to be able to capture the essential physics of the problem. We can quote many examples, where a simple physical model would have saved a lot of subsequent difficulties in system identification; e.g.

(1) In the practical case study referred to in the introduction, elementary physics would have immediately indicated that a simple model containing a time delay plus pure integrator would be a good starting point. (We have deliberately not given details to avoid embarrassing those who did the identification experiment)

(2) In another case study conducted by one of the authors, the associated industrial partners had spent significant effort in developing a large thermodynamic-hydrodynamic model of the process. Yet this model failed to explain key phenomenum of interest. It took considerable time to realize that the massive physical model did not cover an essential part of the physics, namely a non-smooth nonlinearity (slip stick friction in a control valve.)

Our recommendation is therefore to always begin with simple physical reasoning to suggest a model structure. This will typically take the form of a set of ordinary differential equations (possibly nonlinear and with time delays).

\subsection{Which operator?}

The most common operator in use for discrete time models is the shift operator. However, this operator can lead to robustness issues. This is because:

(1) the parameters typically lack physical significance,

(2) the models are usually associated with nontrivial numerical problems. (The source of these difficulties is that a near perfect model, with fast sampling, is invariably $y_{t+1}=y_{t}$ ),

(3) it is difficult to subsequently change the sampling period once a shift operator model has been obtained.

By way of illustration of the above difficulties, we point to the following two continuous time second order systems expressed in terms of the Laplace Transform variable $s$ :

$$
\begin{aligned}
& G_{1}(s)=\frac{1}{s^{2}+s+0.25}=\frac{1}{(s+0.5)^{2}} \\
& G_{2}(s)=\frac{1}{s^{2}+s-0.75}=\frac{1}{(s+1.5)(s-0.5)}
\end{aligned}
$$

The corresponding discrete time models, using a Zero Order Hold $(\mathrm{ZOH})$ with $\Delta=0.1$ are given by (in terms of the Zeta transform variable $z$ ):

$$
\begin{aligned}
G_{1}(z) & =\frac{0.048(z+0.9792)}{z^{2}-1.9025 z+0.9048} \\
G_{2}(z) & =\frac{0.048(z+0.9792)}{z^{2}-1.9120 z+0.9048}
\end{aligned}
$$

It may surprise the reader that learn that these two models exhibit very different behaviour. Indeed, (4) is stable whilst (5) is unstable. Yet, the only difference in this two discrete time models is a subtle (1\%) change in one of the coefficients!. However, in its original form (the continuous time), we clearly see that the one of the coefficients differs by $400 \%$ !

More will be said about this example later.

Our recommendation is to either use continuous time descriptions or delta operator based models. The latter has a close connection to continuous models as we show in section 5 .

\section{CHOICE OF SAMPLING STRATEGY}

The next step is to choose a suitable sample period. A simple rule of thumb is to sample as fast as possible and certainly ten times faster than the dynamics of interest.

One important point that is sometimes overlooked is that sampling should always be preceded by lowpass (anti-aliasing) filtering to avoid folding of high frequency noise back into the bandwidth of interest.

With high speed electro-mechanical systems this filtering is easily carried out with analogue filters. However, filtering is more difficult for systems with long time constants (as are typical for chemical process models). In the latter case, anti-alias filtering can be performed digitally by sampling at a higher rate than finally needed and then low pass filtering via digital filtering techniques. (Of course, there will also be an analogue anti-aliasing filter at the fast sampling rate)

Note that, for robustness reasons, anti-aliasing filtering should be conducted well above the maximum frequency of interest to avoid introducing phase shifts or other contaminations in the range of interest.

\section{SAMPLED DATA FOR CONTINUOUS TIME MODELS}

We have argued in section 4 that one should choose sampling periods which are relatively small compared 
to the dynamics of interest. In this case, one can readily obtain a discrete time model by replacing derivatives in a continuous time model by divided differences. Thus, if we let ${ }^{3} \delta=\frac{q-1}{\Delta}$, then the approximate (derivative replacement) discrete time models corresponding to (4), (5) are

$$
\begin{aligned}
& {\left[\delta^{2}+\delta+0.25\right] y_{t}=u_{t}} \\
& {\left[\delta^{2}+\delta-0.75\right] y_{t}=u_{t}}
\end{aligned}
$$

We see that these models inherit the large parameter difference seen in the continuous model. This suggest that nominal properties will be much improved in the delta form compared with shift operator models. For example, it can be shown that the conditioning number for the least squares parameter estimation goes to $\infty$ (as $\Delta \rightarrow 0$ ) in shift form whereas it goes to 1 in delta form (Middleton and Goodwin, 1990) (see also Goodwin et al. (2007) regarding relative errors due to zero dynamics.)

There has been some reluctance to use Delta domain models because it is (incorrectly) believed that difference operation will be sensitive to noise. However, Delta models simply amount to a linear reparameterization and thus only affect numerical issues. Indeed, the delta operator makes explicit the dependence on differences which is implicit (but alas hidden) in shift operator models.

A word of caution however is that simple derivative replacement models will give a poor description if used in the vicinity of the sampling frequency. This is because sampling inevitably involves folding (i.e. aliasing) of high frequency components back onto the range $(0, \pi / \Delta)$ in the frequency domain.

For example if we assume that the continuous-time system frequency response $G(\mathrm{j} \omega)$ goes to zero as $|\omega| \rightarrow \infty$, then the corresponding discrete-time frequency response converges as follows:

$$
\begin{aligned}
& \lim _{\Delta \rightarrow 0} G_{q}\left(e^{\mathrm{j} \omega \Delta}\right)= \\
& \lim _{\Delta \rightarrow 0} \sum_{\ell=-\infty}^{\infty}\left[\frac{\left(1-e^{-s \Delta}\right)}{s \Delta} G(s)\right]_{s=\mathrm{j} \omega+\mathrm{j} \frac{2 \pi}{\Delta} \ell}
\end{aligned}
$$

The impact of the folding described in (8) is illustrated in Figure 2. This figure shows a comparison of the Bode magnitude diagrams corresponding to a second order system and the exact sampled-data model obtained for different sampling frequencies.

The figure clearly illustrates the fact that, no matter how fast we sample, there is always a difference (near the folding frequency) between the continuous-time model and the discretised models.

A consequence of the folding of high frequency dynamics back onto lower frequencies is that additional

${ }^{3} \Delta$ : sampling period.
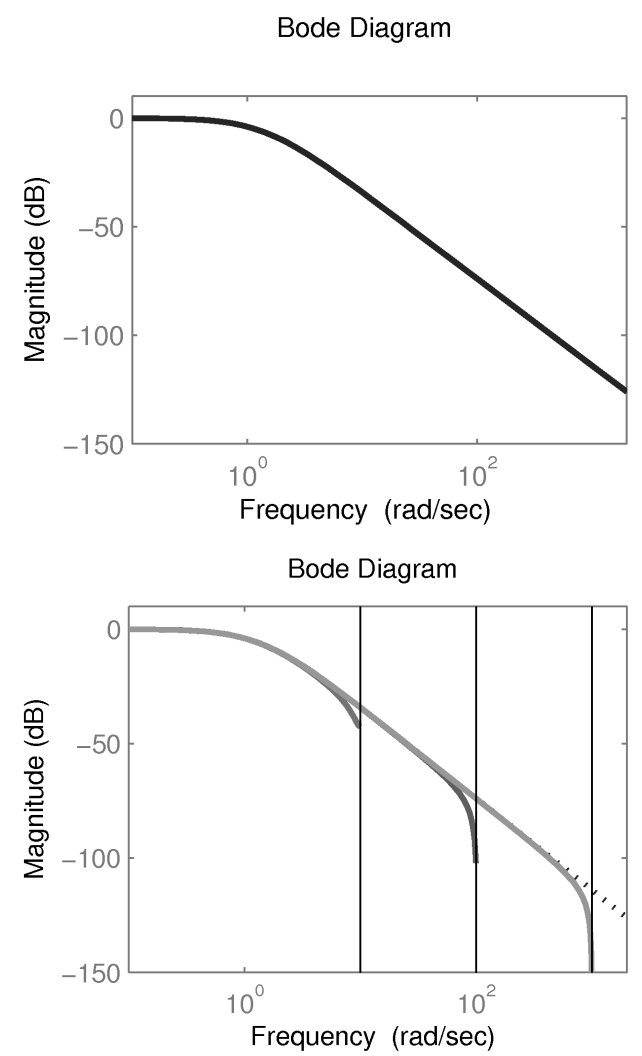

Fig. 2. Continuous- and discrete-time frequency response magnitudes (vertical lines denote folding frequency of $\pi / \Delta)$.

zero dynamics are introduced into the corresponding sampled data model. Thus, for example (6), (7) are more accurately described as follows (for zero order hold input)

$$
\begin{aligned}
& {\left[\delta^{2}+\delta+0.25\right] y_{t}=[\beta \delta+1] u_{t}} \\
& {\left[\delta^{2}+\delta-0.75\right] y_{t}=[\gamma \delta+1] u_{t}}
\end{aligned}
$$

This additional zero corresponds to the asymptotic sampling zero at $z=-1$ in the discrete time models (4) and (5).

Moreover, provided one samples relatively quickly (say ten times the transients of interest), then $\beta$ and $\gamma$ in (9) and (10) can be approximately fixed at the asymptotic value of $0.5 \Delta$ (Feuer and Goodwin, 1996; Goodwin et al., 2007).

The above ideas can be readily generalized. Indeed, there exists a comprehensive theory describing additional sampling zeros of the type shown in (9) and (10). Indeed, for small $\Delta$, it can be shown that, for a system of relative degree $r$, then $(r-1)$ additional sampling zeros appear which asymptotically tend to the roots of the Euler Frobenius polynomials (see e.g. Åström et al. (1984), Weller et al. (2001), Yuz (2005)).

This leads to an interesting follow up robustness question, namely, Should we incorporate the sampling zeros in our model for identification purposes?. Certainly, if one wishes to obtain small (relative) model errors in the vicinity of the sampling frequency, then 
one has no choice but to include the sampling zeros (Yuz and Goodwin, 2007).

This is illustrated in the following example

Example 5.1. Consider a second order linear system:

$$
G_{o}(s)=\frac{\beta_{0}}{s^{2}+\alpha_{1} s+\alpha_{0}}
$$

where the continuous-time parameters are given by $\alpha_{1}=3, \alpha_{0}=2, \beta_{0}=2$. System identification was carried out assuming three different model structures:

SDRM: Simple Derivative Replacement Model. This corresponds to the structure, where continuous-time derivatives are simply replaced by divided differences.

MIFZ: Model Including Fixed Zero. This model considers the presence of the asymptotic zeros.

MIPZ: Model Including Parameterised Zero. This model also includes a sampling zero, whose location has to be estimated.

The three discrete-time models can be represented in terms of the $\delta$ operator as:

$$
G_{\delta}(\gamma)=\frac{B_{\delta}(\gamma)}{\gamma^{2}+\alpha_{1} \gamma+\alpha_{0}}
$$

where:

$$
B_{\delta}(\gamma)=\left\{\begin{array}{lr}
\beta_{0} & (\mathrm{SDRM}) \\
\beta_{0}\left(1+\frac{\Delta}{2} \gamma\right) & (\mathrm{MIFZ}) \\
\beta_{0}+\beta_{1} \gamma & (\mathrm{MIPZ})
\end{array}\right.
$$

We use a sampling period $\Delta=\pi / 100[\mathrm{~s}]$ and choose the input $u_{k}$ to be a random Gaussian sequence of unit variance. Note that the output sequence $y_{k}=y(k \Delta)$ can be obtained by either simulating the continuoustime system and sampling its output, or, alternatively, by simulating the exact sampled-data model in discrete-time. Also note that the data is free of any measurement noise.

The parameters are estimated in such a way to minimise the equation error cost function:

$$
J(\theta)=\frac{1}{N} \sum_{k=0}^{N-1} e_{k}(\theta)^{2}=\frac{1}{N} \sum_{k=0}^{N-1}\left(\delta^{2} y_{k}-\phi_{k}^{T} \theta\right)^{2}
$$

where:

$$
\phi_{k}=\left\{\begin{array}{l}
{\left[-\delta y_{k},-y_{k}, u_{k}\right]^{T}} \\
{\left[-\delta y_{k},-y_{k},\left(1+\frac{\Delta}{2} \delta\right) u_{k}\right]^{T}} \\
{\left[-\delta y_{k},-y_{k}, \delta u_{k}, u_{k}\right]^{T}}
\end{array}\right.
$$

and

$$
\theta=\left\{\begin{array}{lr}
{\left[\alpha_{1}, \alpha_{0}, \beta_{0}\right]^{T}} & (\mathrm{SDRM}) \\
{\left[\alpha_{1}, \alpha_{0}, \beta_{0}\right]^{T}} & (\mathrm{MIFZ}) \\
{\left[\alpha_{1}, \alpha_{0}, \beta_{1}, \beta_{0}\right]^{T}} & (\mathrm{MIPZ})
\end{array}\right.
$$

Note that in this example we are estimating the parameters using unfiltered "equation errors". In practice one should not do this as it is generally extremely non robust. The reason is that equation error models emphasize fitting at high frequencies and are thus very sensitive to high frequency model errors including the issue of having the correct sampling zeros. To explain the origin of the difficulty, consider a general transfer function model of the form:

$$
A_{o}\left(q^{-1}\right) y_{t}=B_{o}\left(q^{-1}\right) u_{t}+w_{t}
$$

where $w_{t}$ is zero mean white noise with variance $\sigma_{w}^{2}$.

Using Parseval's Theorem it is readily seen (Ljung, 1999) that the equation error cost function tends to

$$
J=\int|A|^{2}\left|\left(G_{o}-G\right) \Phi_{u}\right|^{2} d \omega+\int\left|\frac{A}{A_{o}}\right|^{2} \sigma_{w}^{2} d \omega
$$

where $\Phi_{u}, G_{o}=\frac{B_{o}}{A_{o}}$, and $G=\frac{B}{A}$ are the input spectrum ,the "true" system and the model respectively. Now $A\left(q^{-1}\right)$ is, in general, high pass. Thus, we see that the estimator fits the error between $G_{o}$ and $G$ emphasizing high frequencies. Consequently, if one wants to use an equation error estimator then one should always use an appropriate filter, $E\left(q^{-1}\right)$, to focus the fit on the frequency range of interest. With pre-filtering of $y_{t}$ and $u_{t}$ by $E\left(q^{-1}\right)$ the cost function becomes

$$
J=\int\left|\frac{A}{E}\right|^{2}\left|\left(G_{o}-G\right) \Phi_{u}\right|^{2} d \omega+\int\left|\frac{A}{E A_{o}}\right|^{2} \sigma_{w}^{2} d \omega
$$

The filter $E\left(q^{-1}\right)$ can thus focus the estimator in the frequency range of interest. In the remainder of this example we will continue to use unfiltered equation errors so as to emphasize the importance of using the correct sampling zeros if a model with high fidelity at high frequency is desired.

Table 1 shows the estimation results. Note that the system considered is linear, thus, the exact discretetime parameters (exact DT) can be computed for the given sampling period. These are also given in Table 1.

We can see that, while both models incorporating a sampling zero (MIFZ and MIPZ) are able to recover the continuous-time parameters, when using SDRM the estimate $\hat{\beta}_{0}$ is clearly biased (by a factor of almost $2: 1)$.

Table 1. Parameter estimates for a linear system

\begin{tabular}{|c|c|c|c|c|c|}
\hline & \multicolumn{2}{|c|}{ Parameters } & \multicolumn{3}{|c|}{ Estimates } \\
\hline & CT & Exact DT & SDRM & MIFZ & MIPZ \\
\hline$\alpha_{1}$ & 3 & 2.923 & 2.8804 & 2.9471 & 2.9229 \\
$\alpha_{0}$ & 2 & 1.908 & 1.9420 & 1.9090 & 1.9083 \\
$\beta_{1}$ & - & 0.0305 & - & $\frac{\beta_{0} \Delta}{2}=0.03$ & 0.0304 \\
$\beta_{0}$ & 2 & 1.908 & 0.9777 & 1.9090 & 1.9083 \\
\hline
\end{tabular}

The result in the previous example may be surprising since, even though the SDRM converges to the continuous-time system as the sampling period goes 
to zero, the estimate $\hat{\beta}_{0}$ does not converge to the underlying continuous-time parameter. This estimate is asymptotically biased (Yuz, 2005; Yuz and Goodwin, 2007). Specifically, we see that $\beta_{0}$ is incorrectly estimated by a factor of 2 by the SDRM. This illustrates the impact of not considering sampling effects on the sampled-data models used for continuous-time system identification.

The above discussion and example suggest that one cannot, in general, ignore the effect of folding in sampled data models. Indeed, one should include the sampling zero dynamics which result from the folding of high frequency components back into the range $(0, \pi / \Delta)$.

These ideas can also be extended to the nonlinear case (Yuz and Goodwin, 2006). However, there is a further robustness issue here. Specifically, the high frequency components that are folded back by sampling are likely to be ill-defined or non-stationary in practice. Hence, it seems desirable to not place too much confidence in these folded artifacts. For example, the asymptotic sampling zero of $(0.5 \Delta \delta+1)$ described in relation to (9), (10) assumes that the input is generated by a zero order hold and that no undermodelled poles or zeros lie above $\pi / \Delta$. If these assumptions do not hold then this is clearly a source of problems (Yuz and Goodwin, 2007).

\section{LIMITED BANDWIDTH ESTIMATION}

Identification procedures can be robustified to the effects of high frequency folding (including the presence of sampling zeros and anti-aliasing filters) by simply avoiding estimators which focus on frequencies near the folding frequency. This can be readily achieved in the frequency domain (for linear systems) or time domain (for linear and/or nonlinear systems).

\subsection{Restricted bandwidth estimation: The frequency domain}

One can readily perform robust Frequency Domain Maximum Likelihood (FDML) estimation for linear models. The core idea is to convert the data to the frequency domain and then carry out the identification over a limited range of frequencies. Note, however, that one needs to carefully define the likelihood function in this case. For example, the following result (for the scalar case, the result has been derived in (Ljung, 1993), while the multi-variable case is considered in (McKelvey and Ljung, 1997)):

Lemma 1. Assume a given set of input-output data $\left\{u_{k}=u(k \Delta), y_{k}=y(k \Delta)\right\}, k=0 \ldots N$, is generated by the exact discrete-time model:

$$
y_{k}=G_{q}(q, \theta) u_{k}+H_{q}(q, \theta) v_{k}
$$

where $v_{k}$ is Gaussian discrete-time white noise (DTWN) sequence, $v_{k} \sim \mathcal{N}\left(0, \sigma_{w}^{2}\right)$.

The data is transformed to the frequency domain yielding the discrete Fourier transforms $U_{\ell}$ and $Y_{\ell}$ of the input and output sequences, respectively.

Then the maximum likelihood estimate of $\theta$, when considering frequency components up to $\omega_{\max }$, is given by:

$$
\hat{\theta}_{M L}=\arg \min _{\theta} L(\theta)
$$

where $L(\theta)$ is the negative logarithm of the likelihood function of the data given $\theta$, i.e.

$$
\begin{aligned}
L(\theta)= & -\log p\left(Y_{0}, \ldots, Y_{n_{\max }} \mid \theta\right) \\
= & \sum_{\ell=0}^{n_{\max }} \frac{\left|Y_{\ell}-G_{q}\left(e^{\mathrm{j} \omega_{\ell} \Delta}, \theta\right) U_{\ell}\right|^{2}}{\lambda_{v}^{2}\left|H_{q}\left(e^{\mathrm{j} \omega_{\ell} \Delta}, \theta\right)\right|^{2}} \\
& +\log \left(\pi \lambda_{v}^{2}\left|H_{q}\left(e^{\mathrm{j} \omega_{\ell} \Delta}, \theta\right)\right|^{2}\right)
\end{aligned}
$$

where $\lambda_{v}^{2}=\Delta N \sigma_{v}^{2}$, and $n_{\max }$ is the index associated with $\omega_{\max }$.

PROOF. Equation (19) can be expressed in the frequency domain as:

$$
Y_{\ell}=G_{q}\left(e^{\mathrm{j} \omega_{\ell} \Delta}, \theta\right) U_{\ell}+H_{q}\left(e^{\mathrm{j} \omega_{\ell} \Delta}, \theta\right) V_{\ell}
$$

where $Y_{\ell}, U_{\ell}$, and $V_{\ell}$ are scaled discrete Fourier transforms (DFT) (Middleton and Goodwin, 1990; Feuer and Goodwin, 1996), e.g.

$$
Y_{\ell}=Y\left(e^{\mathrm{j} \omega_{\ell} \Delta}\right)=\Delta \sum_{k=0}^{N-1} y_{k} e^{-\mathrm{j} \omega_{\ell} k \Delta} \quad, \omega_{\ell}=\frac{2 \pi}{\Delta} \frac{\ell}{N}
$$

Assuming that the DTWN sequence $v_{k} \sim \mathcal{N}\left(0, \sigma_{w}^{2}\right)$, then $V_{\ell}$ are (asymptotically) independent ${ }^{4}$ and have a circular complex Gaussian distribution (Brillinger, 1974; Brillinger, 1981). Thus, the frequency domain noise sequence $V_{\ell}$ has zero mean and variance $\lambda_{v}^{2}=$ $\Delta N \sigma_{v}^{2}$. We therefore see that $Y_{\ell}$ is also complex Gaussian and satisfies:

$$
Y_{\ell} \sim \mathcal{N}\left(G_{q}\left(e^{\mathrm{j} \omega_{\ell} \Delta}, \theta\right) U_{\ell}, \lambda_{v}^{2}\left|H_{q}\left(e^{\mathrm{j} \omega_{\ell} \Delta}, \theta\right)\right|^{2}\right)
$$

The corresponding probability density function is given by:

$$
\begin{aligned}
p\left(Y_{\ell}\right)= & \frac{1}{\pi \lambda_{v}^{2}\left|H_{q}\left(e^{\mathrm{j} \omega_{\ell} \Delta}, \theta\right)\right|^{2}} \\
& \exp \left\{-\frac{\left|Y_{\ell}-G_{q}\left(e^{\mathrm{j} \omega_{\ell} \Delta}, \theta\right) U_{\ell}\right|^{2}}{\lambda_{v}^{2}\left|H_{q}\left(e^{\mathrm{j} \omega_{\ell} \Delta}, \theta\right)\right|^{2}}\right\}
\end{aligned}
$$

If we consider the elements $Y_{\ell}$ within a limitedbandwidth, i.e. up to some maximum frequency $\omega_{\max }$ indexed by $n_{\max }$ with $\omega_{\max }=\omega_{s} \frac{n_{\max }}{N} \leq \frac{\omega_{s}}{2}$, the appropriate log-likelihood function is given by:

\footnotetext{
4 A simple rule is stationarity in the time domain implies independence in the frequency domain
} 


$$
\begin{aligned}
L(\theta)= & -\log p\left(Y_{0}, \ldots, Y_{n_{\max }} \mid \theta\right)=-\log \prod_{\ell=0}^{n_{\max }} p\left(Y_{\ell}\right) \\
= & \sum_{\ell=0}^{n_{\max }} \frac{\left|Y_{\ell}-G_{q}\left(e^{\mathrm{j} \omega_{\ell} \Delta}, \theta\right) U_{\ell}\right|^{2}}{\lambda_{v}^{2}\left|H_{q}\left(e^{\mathrm{j} \omega_{\ell} \Delta}, \theta\right)\right|^{2}} \\
& +\log \left(\pi \lambda_{v}^{2}\left|H_{q}\left(e^{\mathrm{j} \omega_{\ell} \Delta}, \theta\right)\right|^{2}\right)
\end{aligned}
$$

Remark 2. The logarithmic term must be included in the log-likelihood function since this plays a key role in obtaining consistent estimates of the true system. This term can be neglected only under very special circumstances e.g. if (Ljung, 1993):

- The noise model is assumed to be known. In this case $H_{q}$ does not depend on $\theta$ and, thus, plays no role in the minimisation (20); or

- The frequencies $\omega_{\ell}$ are equidistantly distributed over the full frequency range $\left[0, \frac{2 \pi}{\Delta}\right)$. This is equivalent to considering the full bandwidth case in (25), i.e. $n_{\max }=\frac{N}{2}$ (or $N$, because of periodicity). This yields:

$$
\begin{aligned}
& \frac{2 \pi}{N} \sum_{\ell=0}^{N-1} \log \left|H_{q}\left(e^{\mathrm{j} \omega_{\ell} \Delta}, \theta\right)\right|^{2} \\
& \stackrel{N \rightarrow \infty}{\longrightarrow} \int_{0}^{2 \pi} \log \left|H_{q}\left(e^{\mathrm{j} \omega}, \theta\right)\right|^{2} d \omega
\end{aligned}
$$

A standard result from Complex analysis (The Bode integral (Goodwin et al., 2001)) ensures that the last integral is equal to zero for any monic, stable and inversely stable transfer function $H_{q}\left(e^{\mathrm{j} \omega}, \theta\right)$.

Using the cost function (25) it is straightforward to estimate the parameters. Moreover, tests, e.g. reported in (Yuz, 2005; Yuz and Goodwin, 2007), show this to be a robust strategy.

The above ideas can also be extended to closed loop identification (see Pintelon and Schoukens (2005)), and state-space multivariable systems (Agüero et al., 2007a).

\subsection{Restricted bandwidth estimation in the time domain}

Robust limited bandwidth estimation can also be carried out in the time domain. A first point to note in this context is that sampling inevitably involves a folding of high frequency artifacts back into lower frequencies. Thus, sampled data models are always subject to robustness difficulties at frequencies near the sampling frequency.

If one is principally interested in the plant model (as opposed to the noise model), then there is a simple path to obtaining a limited bandwidth model as explained in the following steps:

(1) Say we are ultimately interested in a discrete time model that operates up to frequency $\pi / \Delta$, then choose a sampling period much smaller than $\Delta$, say $\Delta / 20$.

(2) Excite the system up to frequencies well below $20 \pi / \Delta$, say $5 \pi / \Delta$. Note that this will not give us information near the folding frequency nor will it be informative about the sampling zeros. Indeed, it will suffice to simply use a derivative replacement model (i.e. replace derivatives by divided differences with period $\Delta^{\prime}=\Delta / 20$ ).

(3) Next, assuming that the model found in step 2 is actually continuous time, construct an appropriate sampled data model at the required sample period. This means either manually adding the appropriate zero dynamics resulting from folding or simple use Runge-Kutta to solve the continuous model at the required period. Note that this can be done for any period greater than $\Delta$.

Finally, a word of caution. Whilst step (3) will robustly generate the correct sampling zeros (because our original model has been fitted over the frequency range that will be folded back) it is not advisable to ever run control algorithms with a bandwidth that approaches the folding frequency. This is because, at these periods, there can exist significant control robustness issues. This rules out, for example, controllers such as minimum variance control which, inter alia, yield a closed loop bandwidth near the sampling frequency.

\section{ROBUST EXPERIMENT DESIGN}

Having decided on the class of models and a suitable sampling strategy, then the next step is to design a suitable robust experiment. Colleagues from industry often tell us that the only acceptable experiment (to them) is one performed in closed loop since this ensures that all safety and feedback mechanism are in place (see also the example in Section 2). Indeed in extreme circumstances, it is often said that the experiment should not be detectable on the plant output records (at least as far as plant operators are concerned).

This goal can be given a mathematical description by requiring that the plant output satisfy certain production oriented constraints during the experiment. We will argue below that this type of constraint invariably implies that the experiment should be conducted in closed loop.

\subsection{Linear model description}

For simplicity of exposition we will develop the mathematical support for our conclusions based on linear single input single output models. However, one can 
reasonably expect that similar qualitative conclusions hold more generally (i.e. for nonlinear models, etc.)

Thus, consider a single input - single output linear system of the form

$$
\begin{aligned}
\mathcal{S}=\left\{\left(G_{o}, H_{o}\right) \in \mathcal{C}:\right. & \\
& \left.y(t)=G_{o}\left(q^{-1}\right) u(t)+H_{o}\left(q^{-1}\right) w(t)\right\}
\end{aligned}
$$

where $\mathcal{C}$ is the set of causal linear systems, $q^{-1}$ is the unit delay operator and $G_{o}\left(q^{-1}\right)=q^{-d} \bar{G}_{o}\left(q^{-1}\right)$ $\left(\bar{G}(0)=b_{0} \neq 0, d \in \mathbb{N}\right)^{5}$ and $\{w(t)\}$ is zero mean white noise sequence with variance $E\left\{w(t)^{2}\right\}=\sigma_{w}^{2}$ (note that $\sigma_{w}^{2}$ is also the noise spectral density). We take $H_{o}\left(q^{-1}\right)$ to be the stable minimum phase spectral factor, and $H_{o}(0)=1$.

We consider Box-Jenkins models of the form $G\left(q^{-1}, \theta\right)=$ $G\left(q^{-1}, \rho\right), H\left(q^{-1}, \theta\right)=H\left(q^{-1}, \eta\right)$ where $\theta=\left[\begin{array}{l}\rho \\ \eta\end{array}\right]$. Notice that the sub-parameter vector $\rho$ refers only to $G\left(q^{-1}\right)$.

Under mild conditions, it is well known that when using the Prediction Error Method (PEM) (Goodwin and Payne, 1977, page 99), (Ljung, 1999, page 282):

$$
\sqrt{N}\left(\hat{\theta}_{N}-\theta_{o}\right) \stackrel{d}{\rightarrow} N\left(0, P_{\theta}\right)
$$

where the matrix $P_{\theta}$ (assumed non-singular) is given by:

$$
P_{\theta}=\sigma_{w}^{2}\left[\bar{E}\left\{\Psi\left(t, \theta_{o}\right) \Psi\left(t, \theta_{o}\right)^{T}\right\}\right]^{-1}
$$

and $\Psi(t, \theta)=-\frac{\partial \epsilon(t, \theta)}{\partial \theta}, \epsilon(t, \theta)=H\left(q^{-1}, \theta\right)^{-1}[y(t)-$ $\left.G\left(q^{-1}, \theta\right) u(t)\right]$.

The covariance of the parameter $\hat{\theta}_{N}$ is usually approximated as $\operatorname{cov}\left\{\hat{\theta}_{N}\right\} \approx \frac{1}{N} P_{\theta}$ for experiment design (Ljung, 1999, Chapter 9) ( $N$ is the number of data points).

\subsection{Information matrix for BJ models}

It is well known (Ljung, 1999) that, for BJ models, the following is satisfied:

$$
\Psi\left(t, \theta_{o}\right)=\left[\begin{array}{l}
H_{o}\left(q^{-1}\right)^{-1} \Lambda_{\rho_{o}}\left(q^{-1}\right) u(t) \\
H_{o}\left(q^{-1}\right)^{-1} \Lambda_{\eta_{o}}\left(q^{-1}\right) w(t)
\end{array}\right]
$$

where

$$
\begin{aligned}
& \Lambda_{\rho_{o}}\left(q^{-1}\right)=\left.\frac{\partial G\left(q^{-1}, \rho\right)}{\partial \rho}\right|_{\rho=\rho_{o}} \\
& \Lambda_{\eta_{o}}\left(q^{-1}\right)=\left.\frac{\partial H\left(q^{-1}, \eta\right)}{\partial \eta}\right|_{\eta=\eta_{o}}
\end{aligned}
$$

The information matrix for the full parameter vector is given by:

$$
M_{\theta}=N P_{\theta}^{-1}=\frac{N}{\sigma_{w}^{2}} \bar{E}\left\{\Psi\left(t, \theta_{o}\right) \Psi\left(t, \theta_{o}\right)^{T}\right\}
$$

\footnotetext{
${ }^{5}$ We take $\bar{G}_{o}\left(q^{-1}\right)=\frac{\bar{B}_{o}\left(q^{-1}\right)}{A_{o}\left(q^{-1}\right)}$.
}

This can also be re-written using Parseval's Theorem as ${ }^{6} 7$ (Ljung, 1999, page 291):

$$
M_{\theta}=\frac{N}{\sigma_{w}^{2}} \frac{1}{2 \pi}\left[\begin{array}{cc}
A & B \\
B^{T} & D
\end{array}\right]
$$

where

$$
\begin{array}{r}
A=\int G_{1} G_{1}^{H} \Phi_{u}, B=\int G_{1} G_{2}^{H} \Phi_{u w}, \\
D=\int G_{2} G_{2}^{H} \sigma_{w}^{2}, G_{1}=H_{o}^{-1} \Lambda_{\rho_{o}}, G_{2}=H_{o}^{-1} \Lambda_{\eta_{o}}
\end{array}
$$

From (30) and utilizing standard matrix algebra we have that the inverse of the covariance for $\rho$ and $\eta$ are given by:

$$
\begin{aligned}
& \frac{2 \pi \sigma_{w}^{2}}{N} P_{\rho}^{-1}=\int G_{1} G_{1}^{H} \Phi_{u}-\beta\left(\Phi_{u w}\right) \\
& \frac{2 \pi \sigma_{w}^{2}}{N} P_{\eta}^{-1}=\int G_{2} G_{2}^{H} \sigma_{w}^{2}-\gamma\left(\Phi_{u w}\right)
\end{aligned}
$$

where

$$
\beta\left(\Phi_{u w}\right)=B D^{-1} B^{T}, \quad \gamma\left(\Phi_{u w}\right)=B^{T} A^{-1} B
$$

Remark 3. Notice that $\beta\left(\Phi_{u w}\right) \geq 0$ since $\int G_{2} G_{2}^{H} \sigma_{w}^{2}$ (and its inverse) is a positive definite matrix. Similarly, $\gamma\left(\Phi_{u w}\right) \geq 0$ since $\int G_{1} G_{1}^{H} \Phi_{u}$ (and its inverse) is a positive definite matrix.

For future use we next obtain bounds for $P_{\rho}^{-1}$.

Lemma 4. The inverse of the covariance for $\rho$ (for any experiment $\xi$ ) is bounded as follows: $\frac{2 \pi \sigma_{w}^{2}}{N} P_{\rho}^{-1}\{\xi\} \geq$ $\int G_{1} G_{1}^{H}\left[\Phi_{u}-\frac{\left|\Phi_{u w}\right|^{2}}{\sigma_{w}^{2}}\right]$. Moreover, equality holds if and only if there is a non-frequency dependent matrix $\Gamma$ (of appropriate dimensions) such that $G_{1} \Phi_{u w}=$ $\Gamma G_{2}$.

PROOF. This is a direct consequence of the Cauchy Schwarz inequality (see (Agüero and Goodwin, 2006), (Agüero and Goodwin, 2007) for details).

Our goal in the sequel will be to compare open loop and closed loop experiments. We will use $\Xi_{o l}$ and $\Xi_{c l}$ to denote open and closed loop experiments respectively. We define these classes below.

Consider a general class of experiments carried out with any linear time invariant feedback control law of the form

\footnotetext{
6 Here, and in the sequel, we omit the limits of integration and the integration variable. Unless, otherwise stated the limits of integration are from $-\pi$ to $\pi$, and the integration variable is $\omega$.

$7 \mathrm{We}$ use the following notation: $\Phi_{x y}=$ $\sum_{\tau=-\infty}^{\infty} R_{x y}(\tau) e^{-j \omega \tau}$, and $R_{x y}(\tau)=\bar{E}\{x(t) y(t-$ $\tau)\}=\frac{1}{2 \pi} \int \Phi_{x y} e^{j \omega \tau} d w$ for any pair of signals $x(t)$ and $y(t)$. We also use $\Phi_{x}=\Phi_{x x}$.
} 


$$
u(t)=F_{o}\left(q^{-1}\right) r(t)-C_{o}\left(q^{-1}\right) y(t)
$$

where $r(t)$ is a reference signal. This class includes open loop experiments where we take $C_{o}\left(q^{-1}\right)=0$.

Under the control law (33), the closed loop satisfies

$$
\begin{aligned}
& y(t)=G_{o} F_{o} S_{o} r(t)+S_{o} H_{o} w(t) \\
& u(t)=F_{o} S_{o} r(t)-C_{o} H_{o} S_{o} w(t)
\end{aligned}
$$

where $S_{o}\left(q^{-1}\right)$ is the sensitivity function given by (Goodwin et al., 2001, page 125):

$$
S_{o}\left(q^{-1}\right)=\frac{1}{1+G_{o}\left(q^{-1}\right) C_{o}\left(q^{-1}\right)}
$$

The corresponding output, input and cross spectrum are given by

$$
\begin{aligned}
\Phi_{y} & =\left|G_{o} F_{o} S_{o}\right|^{2} \Phi_{r}+\left|S_{o} H_{o}\right|^{2} \sigma_{w}^{2} \\
\Phi_{u} & =\left|F_{o} S_{o}\right|^{2} \Phi_{r}+\left|C_{o} H_{o} S_{o}\right|^{2} \sigma_{w}^{2} \\
\Phi_{u w} & =-C_{o} H_{o} S_{o} \sigma_{w}^{2}
\end{aligned}
$$

We can then define the classes of experiments of interest as follows:

\section{Definition 1.}

$$
\begin{aligned}
\text { Open loop: } \Xi_{o l} & =\left\{\xi \in \Xi: C_{o}\left(q^{-1}\right)=0\right\} \\
\text { Closed loop: } \Xi_{c l} & =\left\{\xi \in \Xi: C_{o}\left(q^{-1}\right) \neq 0\right\}
\end{aligned}
$$

With the above background we can now define what we mean by a good experiment. Specifically:

Definition 2. The class $\Xi_{c l}$ of experiments is said to be better (not worse) than the class $\Xi_{o l}$ if and only if $\forall \xi_{c} \in \Xi_{c l}, \exists \xi_{o} \in \Xi_{o l}$ such that ${ }^{8}$

$$
P_{\rho}^{-1}\left\{\xi_{c}\right\}>P_{\rho}^{-1}\left\{\xi_{o}\right\} \quad\left(P_{\rho}^{-1}\left\{\xi_{c}\right\} \geq P_{\rho}^{-1}\left\{\xi_{o}\right\}\right)
$$

The above definition uses a strong notion of optimality called Loewner optimality (Pukelsheim, 1993, chapter 4) due to the association with the ordering of symmetric matrices called The Loewner partial ordering (see e.g. (Siotani, 1967)). This definition can be extended to define a partial ordering (for the reduced vector $\rho$ ) amongst experiments in the sense that (Kiefer, 1959)

$$
\xi_{1} \stackrel{\rho}{\succeq} \xi_{2} \Leftrightarrow P_{\rho}^{-1}\left\{\xi_{1}\right\} \geq P_{\rho}^{-1}\left\{\xi_{2}\right\} \quad \xi_{1}, \xi_{2} \in \Xi
$$

where $\rho$ is the vector of parameters of interest and $\Xi$ is the allowable set of experiments. When strict inequality holds in (37) we write $\xi_{1} \stackrel{\rho}{\succ} \xi_{2}$.

Remark 5. Notice that $\xi_{1} \stackrel{\rho}{\succeq} \xi_{2}$ implies that $\xi_{1}$ is preferable under any order preserving (isotonic) criterion such as $\operatorname{det}\left\{P_{\theta}\right\}, \lambda_{\max }\left\{P_{\theta}\right\}$, trace $\left\{P_{\theta}\right\}$, etc. $\nabla \nabla \nabla$

\footnotetext{
$8 A \geq B$ and $A>B$ signify $A-B$ positive semidefinite and positive definite respectively.
}

\subsection{Open v/s Closed Loop}

To obtain a meaningful design problem, it is necessary to place constraints on the allowable set of experiments. Based on our stated goal that the experiment should have minimal impact on nominal production, we require that the experiment satisfy an output power constraint i.e. we require that

$$
\mathcal{P}_{y}: \int \Phi_{y} \leq K
$$

Note that this requirement is heuristically related to keeping the perceived output variations small during the experiment.

The constant $K$ is assumed to be strictly greater than the minimum achievable output variance. In order to find an optimal solution we use the scalar function $J\left(P_{\rho}^{-1}\right)$ which is any isotonic (order preserving) function.

Our key conclusion is then summarized in the following result (Agüero and Goodwin, 2006; Agüero and Goodwin, 2007):

Theorem 6. For the system described in equation (27) and provided that a BJ model is used and that the associated minimum variance controller is different from zero then the class of experiments $\Xi_{c l}$ is better than the class of experiments $\Xi_{o l}$. Moreover, for any isotonic scalar design criterion, $J(\cdot)$ for $\rho$, the optimal experiment is necessarily in the class $\Xi_{c l}$.

PROOF. See Appendix.

The above result is very strong since it shows that, if one constrains the output power during an experiment, then one should always perform the experiment in closed loop (for a general class of systems as stated in the Theorem). The proof of this Theorem is based on the construction of a closed loop experiment which is better than any given open loop experiment. Indeed, the closed loop experiment need only to satisfy the following:

$$
\int\left|S_{o} H_{o}\right|^{2}<\int\left|H_{o}\right|^{2}
$$

where $S_{o}$ is the closed loop sensitivity function.

Actually, we can gain a little more insight by examining this latter requirement for the closed loop experiment. What this says is that the (mean square) impact of disturbances during the (closed loop) experiment should be less in closed loop than it would have been had the experiment been performed in open loop. Indeed, the more we can reduce the impact of disturbances on the output, then the greater "room" we make for the output variations caused by the experimental test signal (within the limits imposed by production constraints). This seems heuristically reasonable. 
One might actually believe by examining (39) that the best experiment coincides with making $\int\left|S_{o} H_{o}\right|^{2}$ as small as possible. Indeed, the controller that makes the output variance as small as possible is commonly known as a minimum variance controller. The design of such a controller depends on having a detailed plant model. However, this introduces a robustness problem since the whole purpose of the experiment is to learn (more) about the plant model. (More will be said on this point later).

However, this line of reasoning is readily resolved (at least heuristically) by utilizing ideas from traditional control theory. Specifically we know that $\left|S_{o}\right|$ can be made small over the frequencies where the relative model error is less than one. Hence, prior knowledge about the system dictates the bandwidth over which we can robustly obtain significant sensitivity reduction in experiment design. Indeed, an iterative design procedure can be employed where one successively fits a model (over a given bandwidth), then uses that model to design a controller to achieve a slightly wider bandwidth and so on. This also is related to the windsurfer approach to adaptive control (Anderson and Kosut, 1991; Lee et al., 1993; Lee et al., 1995; Kosut, 2000).

\subsection{Design of the reference signal}

The key conclusion from Section 7.3 is that, subject to the output variations being constrained, we should design the experiment so that it is conducted in closed loop. We also conclude (see proof of Theorem 6 in the Appendix) that the reference should be injected via $\bar{S}_{o}$ where $\bar{S}_{o} S_{o}$ is all pass. However, so far, we said nothing about the reference signal perturbations themselves. Examination of the proof of Theorem 6 shows that a good option is to use the best "open loop" test signal in conjunction with tight feedback control. Here again we are confronted by a robustness issue, namely the best open loop test signal typically depends on the nature of the system i.e. the very thing that the experiment is aimed at learning.

We are thus lead to consider more heuristic (and practical) issues. Practitioners who carry out experiments often report that step type test signals are good, but often do not excite high frequencies terms adequately. On the other hand random signals such as PRBS are also considered good, but often have wasted energy at high frequencies.

In the frequency domain, step type inputs have power that decays as $1 /(\text { frequency })^{2}\left(1 / f^{2}\right)$ whereas random signals have power that is constant over frequency. The above line of reasoning implies that a signal having power that lies somewhere between $1 / f^{2}$ and a constant might be a good open-loop test signal. This suggests that a test signal with power that decays as $1 / f$ (over a limited bandwidth) could be a good choice. Indeed, recent research has shown that

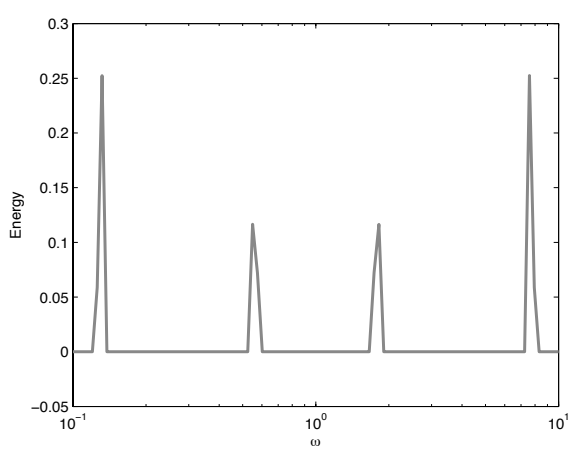

Fig. 3. Discretised spectrum of the robust optimal input for the first order system.

test signals having energy that decays as $1 /(f)$ have remarkable robustness properties in system identification. This claim is illustrated below for a particular example.

As a specific illustration of the properties of (bandlimited) $1 / f$ noise, we refer to an illustrative first order system having transfer function

$$
G(s)=\frac{1}{s / \theta+1}
$$

The optimal nominal (open loop) test signal for identification of this system is well known to be a single sinusoid of frequency $w^{*}=\theta_{o}$ where $\theta_{o}$ is the a-priori estimate of $\theta$.

This is an intuitively pleasing result, i.e. one places the test signal at the (nominal) $3 \mathrm{~dB}$ break point. However, the result reinforces the fundamental robustness difficulty associated with nominal experiment design, namely, the optimal (open loop) test signal depends on the very thing that the experiment is aimed at estimating.

An alternative, robust input design strategy is to assume that the a-priori distribution of $\theta$ is anywhere in a compact set $\Theta$. Then, robust experiment design may be formulated as

$$
\xi=\min _{\xi \in \Xi} \max _{\theta \in \Theta} J(P(\theta), \theta)
$$

where $\xi$ is the experimental conditions (here the test signal), $J(\cdot)$ a suitable scalar function of $P(\theta)$ the parameter variance matrix.

For the one parameter problem (40), we choose $\Theta=$ $\left[\theta_{\min }, \theta_{\max }\right]$ and $J(P(\theta), \theta)$ as the relative error $P(\theta) / \theta^{2}$. For $\theta_{\min }=1$ and $\theta_{\max }=10$.

For the above problem, the following key properties have been established in (Rojas et al., 2007c):

(i) Existence: There exists at least one optimal input.

(ii) Uniqueness: The optimal input is unique, and $\underline{\theta}$ and $\bar{\theta}$ do not belong to the input spectrum. 
(iii) Compact support: Every optimal input should have all its energy inside $[\underline{\theta}, \bar{\theta}]$.

(iv) Finite support: The optimal input has finite support in the frequency domain, and thus can be realised as a finite sum of sinusoids. (Recall the test signal deployed in the motivational example of Section 2).

Figure 3 shows the robust optimal test signal obtained via the cost function (41).

A remarkable property (established in (Rojas et al., $2007 c)$ ) is that bandlimited ' $1 / f$ ' noise, defined by the spectrum

$$
\phi_{u}^{1 / f}(\omega) \triangleq\left\{\begin{array}{ll}
\frac{1 / \omega}{\ln \bar{\omega}-\ln \underline{\omega}}, & \omega \in[\underline{\omega}, \bar{\omega}] \\
0, & \text { otherwise }
\end{array},\right.
$$

is near optimal. Here we take the frequency range as $\underline{\omega}=\underline{\theta}$ and $\bar{\omega}=\bar{\theta}$. In fact, it has been proven that the performance of bandlimited ' $1 / f$ ' noise is (at most) a factor of 2:1 away from the performance of the true robust optimal test signal.

Table 2 compares the achieved performance for different test signals.

Note that the single sinusoid at $\omega=1$ is the nominal test signal if we take the nominal parameter value as the geometric mean of $\theta_{\min }$ and $\theta_{\max }$.

We see from Table 2 that $1 / f$ noise is indeed an excellent input for robust experiment design. This is further supported by recent research reported in (Rojas et al., 2007a).

In practice, it is also desirable to keep the amplitude of the test signal small. Thus, one may be interested in generating binary signals having a (band-limited) $1 / f$ spectrum. Methods for designing such test signals are described in (Rojas et al., 2007b; Agüero et al., 2007b).

\section{ESTIMATION PROCEDURES}

We have argued above that the best experiment (when the ouput variations are constrained) is a closed loop one. This then leads to the obvious follow up question, namely how should we estimate the parameters from closed loop data.

This issue has led to considerable consternation by practitioners. Indeed, some say that closed loop identification is simply impossible. We will show below that, in fact, closed loop identification, when carried out properly, is no more difficult than open loop identification.

Thus, consider the closed loop shown in Figure 4. To illustrate the ideas we assume that the process of interest has the following linear model (extensions to the nonlinear case are also possible):

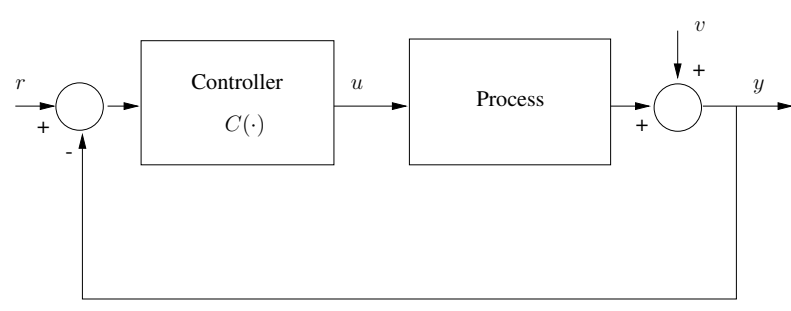

Fig. 4. Closed Loop used in Identification

$$
\begin{aligned}
& y_{t}=G_{o} u_{t}+v_{t} \\
& v_{t}=H_{o} w_{t}
\end{aligned}
$$

where $G_{o}$ and $H_{o}$ are linear transfer functions, and $w_{t}$ is a sequence of independent random variables with zero mean value, variance $\sigma_{w}^{2}$. We also, assume that $y_{t}$ and $u_{t}$ are jointly quasi-stationary, that the model $G$ for $G_{o}$ belongs to the family of models $G(\theta)$, and that $H$ (the model for $H_{o}$ ) belongs to a family of models $H(\theta)$.

The equations describing the system in Figure 4 are

$$
\begin{aligned}
& u_{t}=C\left(q^{-1}\right)\left[r_{t}-y_{t}\right] \\
& y_{t}=G_{o}\left(q^{-1}\right) u_{t}+v_{t} \\
& v_{t}=H_{o}\left(q^{-1}\right) w_{t}
\end{aligned}
$$

where $w_{t}$ is a white noise sequence.

The closed loop system is assumed to be stable.

We further assume that at least one of the following two conditions holds:

(1) There is a delay in both the process and the model $\left(G_{o}(0)=G(0)=0\right)$ and

(2) the true controller is strictly proper $(C(0)=0)$.

Also, we normalize the true noise transfer function $H_{o}\left(q^{-1}\right)$, and the model $H\left(q^{-1}\right)$ by requiring that

$$
H_{o}(0)=H(0)=1
$$

\subsection{Review of results on direct and indirect identification using PEM}

The literature on identification offers two choices for closed loop identification ${ }^{9}$

- Direct: Here one treats $u_{t}$ and $y_{t}$ as if they were in open loop and estimates $G$ directly.

- Indirect: Here the relationship between $y_{t}$ and $r_{t}$ is modeled and then $G_{o}$ is obtained from this model. In the linear case, we use the model

$$
y_{t}=\frac{G_{o} C}{1+G_{o} C} r_{t}+\frac{1}{1+G_{o} C} v_{t}
$$

and then extract an estimate of $G_{o}$. Indeed (48) can be simply thought as a potential model structure with some known parts (the parameters in

\footnotetext{
9 There are some other alternatives in the available literature such as joint input/output identification. However, all of them assume perfect knowledge of the controller. Moreover, in most cases the controller must be linear.
} 
Table 2. Relative Values of Cost for the Different Input Signals

\begin{tabular}{|c|c|}
\hline & $\max _{\theta \in \Theta}\left[\theta^{2} \bar{M}\left(\theta, \phi_{u}\right)\right]^{-1}$ \\
\hline \hline Sinusoid at $\omega=1$ & 7.75 \\
\hline Bandlimited white noise & 12.09 \\
\hline Bandlimited ' $1 / f$ ' noise & 1.43 \\
\hline Min-max optimal input & 1.00 \\
\hline
\end{tabular}

$C$ ) and some parts to be estimated (the parameters in $G_{o}$ ).

Each of the above approaches has advantages and disadvantages. Specifically,

(1) Direct identification is impossible with open loop unstable systems (in Box Jenkins form) since one has no way to ensure that the unstable initial condition response remains bounded. Also, direct identification is sensitive to being able to accurate specification of the noise model. Indeed, we will show below that errors in the noise model lead to bias.

(2) Indirect identification is, on the other hand, sensitive to the fidelity of the controller $C$. Thus, errors in the controller, e.g. due to saturation, will cause bias errors.

Note that indirect identification is equivalent to direct identification of a (closed loop) model having a particular parameterization.

We take a short diversion to review known results (Forssell and Ljung, 1999; Ljung, 1999) on identification via PEM's.

We assume that the system under study has input $\bar{u}_{t}$ and output $y_{t}$. (The specific form of $\bar{u}_{t}$ and its relationship to $u_{t}$ will be described later.) We conceptually model the relationship between $\bar{u}_{t}$ and $y_{t}$ by

$$
y_{t}=G \bar{u}_{t}+H w_{t}
$$

where $w_{t}$ is notionally "white noise" and $G$ and $H$ are (independently) parameterized transfer functions. The PEM typically uses a cost function of the form (Ljung, 1999):

$$
V=\frac{1}{N} \sum_{t=1}^{N} \epsilon_{t}^{2}
$$

where $\epsilon_{t}$ denotes the prediction error given by:

$$
\epsilon_{t}=H^{-1}\left[y_{t}-G \bar{u}_{t}\right]
$$

The following results are standard for PEM identification:

Lemma 7. The cost function $V(\theta)$ converges, almost surely, to

$$
\bar{V}(\theta)=\bar{E}\left\{\epsilon_{t}^{2}\right\}=\frac{1}{2 \pi} \int_{-\pi}^{\pi} \Phi_{\epsilon}(w) d w
$$

PROOF. See (Forssell and Ljung, 1999; Ljung, 1999).
Lemma 8. The prediction error spectrum is given by

$$
\Phi_{\epsilon}=\sigma_{w}^{2}+\frac{1}{|H|^{2}}\left[\begin{array}{ll}
\tilde{G} & \tilde{H}
\end{array}\right] \Phi_{X}\left[\begin{array}{l}
\tilde{G}^{*} \\
\tilde{H}^{*}
\end{array}\right]
$$

where $*$ denotes complex conjugate and

$$
\begin{gathered}
\tilde{G}=G_{o}-G \\
\tilde{H}=H_{o}-H \\
\Phi_{X}=\left[\begin{array}{cc}
\Phi_{\bar{u}} & \Phi_{\bar{u} w} \\
\Phi_{w \bar{u}} & \sigma_{w}^{2}
\end{array}\right]
\end{gathered}
$$

PROOF. See (Forssell and Ljung, 1999; Ljung, 1999)

Using the result (53) it is easy to see that if the spectrum $\Phi_{X}$ is a positive definite matrix for all frequencies, and that the true model $\left(G_{o}\right.$ and $\left.H_{o}\right)$ are contained in the family of models $(G(\theta)$ and $H(\theta))$ a consistent estimate is obtained. This condition has been called an informative experiment (Ljung, 1999; Forssell and Ljung, 1999).

We also have the following result:

Lemma 9. The prediction error spectrum can be written as:

$\Phi_{\epsilon}=\sigma_{w}^{2}+\frac{1}{|H|^{2}}\left[\left|\tilde{G}+\tilde{H} \Phi_{w \bar{u}} \Phi_{\bar{u}}^{-1}\right|^{2} \Phi_{\bar{u}}+|\tilde{H}|^{2} \Phi_{w}^{r}\right]$

where $\tilde{G}, \tilde{H}$ are as in (54), (55), and

$$
\Phi_{w}^{r}=\sigma_{w}^{2}-\Phi_{w \bar{u}} \Phi_{\bar{u}}^{-1} \Phi_{\bar{u} w}
$$

PROOF. See (Forssell and Ljung, 1999; Ljung, 1999).

Using (57), it can be readily seen that, when we have an erroneous noise model, the resulting estimate of $G_{o}$ will have an asymptotic bias given by:

$$
B_{\theta}=\tilde{H} \Phi_{w \bar{u}} \Phi_{\bar{u}}^{-1}
$$

where $\tilde{H}$ is as in (55).

Here, we have assumed that $H$, and $G$ are independently parameterized. This, also covers the case when a fixed noise model is used.

Using equation (59) we see that it is possible to obtain a consistent estimate in the following two cases:

- When a sufficiently rich family of noise models $H(\theta)$ is used such that $H=H_{o}$ is achievable. 
- When the cross-spectrum $\Phi_{w \bar{u}}$ is (near) zero. (This is always the case with open loop data but will generally be false for closed loop data when a direct identification method is used).

Equation (57) also gives insight when the system model is misspecified i.e. when there does not exist a $G$ in the model class such that $\tilde{G}$ can be zero. In this case, minimization of (52) can be viewed as an approximation problem where the "weighted distance" between $G$ and $G_{o}+\tilde{H} \Phi_{w u} \Phi_{u}^{-1}$ is minimized over the given model class.

\section{VIRTUAL CLOSED LOOP IDENTIFICATION}

We have seen in section 8 that both direct and indirect closed loop identification have potential robustness issues. In this section, we describe a procedure that combines the best features of direct and indirect identification.

To explain the idea, consider a hypothetical controller, $\bar{C}$, which is linear and has the transfer function

$$
\bar{C}\left(q^{-1}\right)=\frac{P\left(q^{-1}\right)}{L\left(q^{-1}\right)}
$$

We use a fractional representation and express (60) in the equivalent form:

$$
\bar{C}\left(q^{-1}\right)=\frac{P\left(q^{-1}\right) / E\left(q^{-1}\right)}{L\left(q^{-1}\right) / E\left(q^{-1}\right)}
$$

where $E\left(q^{-1}\right)$ is any stable polynomial. We also write

$$
N\left(q^{-1}\right)=E\left(q^{-1}\right)-L\left(q^{-1}\right)
$$

Next we form a filtered version of the reference signal via the following stable operations on the measured signals $u_{t}$ and $y_{t}$ :

$$
\bar{u}_{t}=u_{t}-\frac{N\left(q^{-1}\right)}{E\left(q^{-1}\right)} u_{t}+\frac{P\left(q^{-1}\right)}{E\left(q^{-1}\right)} y_{t}
$$

We can now use the virtual input, $\bar{u}_{t}$, as a simple mechanism for choosing an identification algorithm that lies anywhere in the range from direct to indirect. To illustrate, we consider two extreme cases.

(1) When $\bar{C}=\frac{P}{L}$ is chosen as the true control law, then

$$
\begin{aligned}
& u_{t}=\frac{P\left(q^{-1}\right)}{L\left(q^{-1}\right)}\left[y_{t}-r_{t}\right] \\
& \bar{u}_{t}=u_{t}-\frac{N}{E} u_{t}+\frac{P}{E} y_{t}
\end{aligned}
$$

Substituting (64) into (65) gives

$$
\begin{aligned}
\bar{u}_{t} & =-\left(1-\frac{N}{E}\right)\left(\frac{P}{L}\right)\left[y_{t}-r_{t}\right]+\frac{P}{E} y_{t} \\
& =-\frac{P}{E}\left[y_{t}-r_{t}\right]+\frac{P}{E} y_{t} \\
& =\frac{P}{E} r_{t}
\end{aligned}
$$

Hence, in this case, $\bar{u}_{t}$ is simply a filtered version of the reference input. Thus, if we use direct identification the system linking $\bar{u}_{t}$ to $y_{t}$, we are, in effect, using indirect identification between a reference signal $\frac{P}{E} r_{t}$ and the output $y_{t}$.

(2) Next, say we choose $E\left(q^{-1}\right)=L\left(q^{-1}\right)$ (i.e. $\left.N\left(q^{-1}\right)=0\right)$ and $P\left(q^{-1}\right)=0$, then from (63) we have that $\bar{u}_{t}=u_{t}$. Hence, in this case, $\bar{u}_{t}$ is simply the true plant input. Thus, fitting a model between $\bar{u}_{t}$ and $y_{t}$ corresponds to the usual direct identification method.

Our principal interest here is when $\bar{C}\left(q^{-1}\right)$ is not the true controller. Indeed, all we require is that $\bar{C}\left(q^{-1}\right)$ stabilizes the system if it were to be applied to the system (even if it is, in fact, not in the real closed loop!).

When $\bar{C}\left(q^{-1}\right)$ is not the true controller, it is still easy to compute the equations linking $\bar{u}_{t}$ to $y_{t}$. Specifically, we have from (63) that $y_{t}$ and $\bar{u}_{t}$ are related by the following Virtual Closed Loop (where we treat $\bar{u}_{t}$ as a given signal).

Plant model: $\quad y_{t}=G\left(q^{-1}\right) u_{t}+v_{t}$

Virtual feedback: $u_{t}=\frac{N\left(q^{-1}\right)}{E\left(q^{-1}\right)} u_{t}-\frac{P\left(q^{-1}\right)}{E\left(q^{-1}\right)} y_{t}+\bar{u}_{t}$

where $\left\{v_{t}\right\}$ denotes the noise sequence and is assumed to satisfy $v_{t}=H\left(q^{-1}\right) w_{t}$ for $\left\{w_{t}\right\}$ a white sequence.

Remark 10. The plant model $G\left(q^{-1}\right)$ in (69) could be unstable, nonlinear etc. However, for (69) to be suitable for identification it is necessary that $\bar{C}\left(q^{-1}\right)$ stabilizes the plant model. $\nabla \nabla \nabla$

Remark 11. Equations in (69) simply represent a particular parameterized model linking $\bar{u}_{t}$ to $y_{t}$ where parts of the model are fixed and known (namely $N\left(q^{-1}\right), E\left(q^{-1}\right)$ and $\left.P\left(q^{-1}\right)\right)$ and parts are unknown (namely the parameters in $G\left(q^{-1}\right)$ ). See (72) below. $\nabla \nabla \nabla$

Finally, one can ask where do we get $\bar{u}_{t}$ from to drive (69). The answer is again provided by (63) i.e.

$$
\bar{u}_{t}=u_{t}-\frac{N\left(q^{-1}\right)}{E\left(q^{-1}\right)} u_{t}+\frac{P\left(q^{-1}\right)}{E\left(q^{-1}\right)} y_{t}
$$

Remark 12. When $\bar{C}\left(q^{-1}\right)$ is not the true controller, then there will exist residual correlations between the noise and $\bar{u}_{t}$. This will, in turn, lead to residual bias errors if the associated noise model is erroneous. However, we can see that one gains the advantages of both direct identification (i,e. $\bar{u}_{t}$ can be treated as a known input into a particularly parameterized plant) and indirect identification can be performed since the virtual controller is exactly known. 
Table 3. Monte-Carlo comparison between VCL (PEM) and Direct (PEM) method for $N=3000$ data points

\begin{tabular}{|c|c|c|}
\hline$\sigma_{w}^{2}=0.1$ & $a$ & $b$ \\
\hline \hline True Values & 0.8 & 0.2 \\
\hline Mean Value VCL Identification & 0.7955 & 0.1990 \\
\hline Mean Value Direct Identification & 0.8634 & 0.3041 \\
\hline Mean Value Indirect Identification & 0.7746 & 0.1515 \\
\hline
\end{tabular}

\subsection{Analysis of the effect of the virtual closed loop on} bias

Our goal in this section is to show that the use of a virtual closed loop has a beneficial effect on estimation accuracy. In particular, we wish to study the effect of the virtual closed loop on the correlation between $w$ and $\bar{u}_{t}$ and hence on the bias expression given in (59).

The key difference between direct identification and virtual closed loop identification is that direct identification is based on the model

$$
y_{t}=G_{o} u_{t}+H_{o} w_{t}
$$

whereas, the virtual closed loop identification is based on (69) which can also be expressed as

$$
y_{t}=\frac{G \frac{E}{L}}{1+G \bar{C}} \bar{u}_{t}+\frac{H}{1+G \bar{C}} w_{t}=T^{y} \bar{u}_{t}+\bar{H} w_{t}
$$

The key problems with (71) are

(1) One cannot run the model $G$ in open loop if it is unstable.

(2) The presence of feedback means that $u_{t}$ and $w_{t}$ are potentially highly correlated which is a source of robustness and bias problems.

On the other hand, the model $\frac{G \frac{E}{L}}{1+G \bar{C}}$ can be stable even if $G$ is unstable. Also, the correlations between $\bar{u}$ and $w_{t}$ are potentially much less than between $u_{t}$ and $w_{t}$. The implications of the above observations are explained in detail in (Agüero and Goodwin, 2004b; Agüero and Goodwin, 2004a; Agüero, 2005). In particular we argue in (Agüero and Goodwin, 2004a; Agüero, 2005), that the bias resulting from virtual closed loop identification has the form

$$
\hat{G}=G_{o} \bar{\lambda}-\bar{C}^{-1}(1-\bar{\lambda})
$$

where

$$
\bar{\lambda}=\frac{1}{1+\kappa C_{\Delta} H_{\Delta}}
$$

where $\kappa$ is a frequency dependent parameter, $C_{\Delta}$ denotes the relative error in the virtual controller i.e. the true controller $C_{l}$ is expressed as

$$
C_{l}=\bar{C}\left(1+C_{\Delta}\right)
$$

and where $H_{\Delta}$ denotes the error in the noise model, i.e.

$$
H_{\Delta}=\bar{H}-\bar{H}_{o}
$$

Also note that $\kappa$ is inversely proportional to the size of the reference signal spectrum.

Remark 13. We see from (73) that the bias in the estimate will be small if any of the following three conditions is satisfied

(1) $H_{\Delta}$ is small (i.e. small noise model errors)

(2) $C_{\Delta}$ is small (i.e. small errors between $\bar{C}$ and the true controller).

(3) The reference signal dominates disturbances.

$\nabla \nabla \nabla$

Thus, the virtual closed loop method achieves the best features of both direct and direct identification.

\subsubsection{Example Consider the following system:}

$$
G_{o}\left(q^{-1}\right)=\frac{b q^{-1}}{1-a q^{-1}}
$$

with $a=0.8$, and $b=0.2$. This system is operated in closed loop with the following nominal linear controller:

$$
C_{l}\left(q^{-1}\right)=\frac{0.3}{1-q^{-1}}
$$

However, the true controller operates in such a fashion that the input signal saturates such that $\left|u_{t}\right| \leq 5$. (Recall the saturating controller used in the motivating example of Section 2). Thus, the true controller, $C$, is actually nonlinear. The reference signal $r_{t}$ is taken to be zero mean Gaussian white noise process of variance $\sigma_{r}^{2}=30$. The noise $w_{t}$ is taken to be zero mean Gaussian white noise process of variance $\sigma_{w}^{2}=$ $0.1\left(\sigma_{v}^{2} \approx 5\right)$. The true noise model is given by

$$
H_{o}\left(q^{-1}\right)=\frac{1}{1+d_{1} q^{-1}+d_{2} q^{-2}+d_{3} q^{-3}+d_{4} q^{-4}}
$$

where $d_{1}=-1.992, d_{2}=2.202, d_{3}=-1.841$, and $d_{4}=0.8941$.

In order to identify the process, we assume (incorrectly) that the noise model, $H$, is an autoregressive AR model of first order. (This is, after all, not unreasonable, since the system is a first order transfer function). We then identify the process by using direct identification, and also by using the virtual closed loop method with the virtual controller equal to the true controller (but without saturation). For the Virtual Closed Loop (VCL) case, we use a PEM to identify the virtual closed loop transfer function. (We use a filter 
$\left.E=1-0.95 q^{-1}\right)$. We then extract the estimate of the open loop model by using the known relationship between the open and closed loop parameters. We carried out 400 Monte-Carlo simulations for 3000 data points. The average models so obtained are presented in table 3 .

We can see that, for this example, the model obtained by using direct identification is not satisfactory - there is a significant model misfit due to a poor noise model. Also, the model found by indirect identification is poor. This is because the controller occasionally saturates. On the other hand, the model obtained by using the virtual closed loop method is extremely close to the true model.

The reason for this improvement is that the use of a virtual closed loop, dramatically reduces the bias due to the reduction in the value of $\beta$. (see equation (73)).

\section{A SECOND INDUSTRIAL EXAMPLE}

Finally, we present a second industrial case study that illustrate some of the issues raised above.

The system is a continuous metal caster which requires very good level control in the mould to ensure that product quality is maintained at a high standard. The system input is the position of a slide gate valve, and the output is the height of molten metal in the mould.

The company had applied non robust identification methods, to data collected during normal operation, to obtain a model of the system for the purpose of improving their control system. Their analysis recommended models of 4th and 5th order. When these models were used to design a controller, very poor control was experienced. The problem was that they were operating in closed loop with no external excitation in the presence of significant output disturbances. Hence, as discussed in previous sections, they were actually identifying a model that was strongly biased toward the negative inverse of the controller. This can be clearly seen in Figure 6 were a Bode magnitude plot of the Empirical Transfer Function Estimate (ETFE) (Ljung, 1999) and $-1 / C$ are presented.

Recognising the problem associated with identification in closed loop, one can then set about ensuring that the bias towards the negative inverse of the controller in minimised. This was accomplished in this case study by adding external excitation to the system such that a reasonably large signal to noise ratio was obtained at the output at a fixed set of frequencies. In this particular case the external signal was added to the controller output (plant input). Figure 5 shows a time slice of both the input and output signals during an experiment where a sum of sinusoids was applied as the external excitation. The Bode magnitude response of the system to the test signal can be seen in Figure 6 as indicated by the $\times$ symbols.


Fig. 5. Plot of input and output signals during the experiment

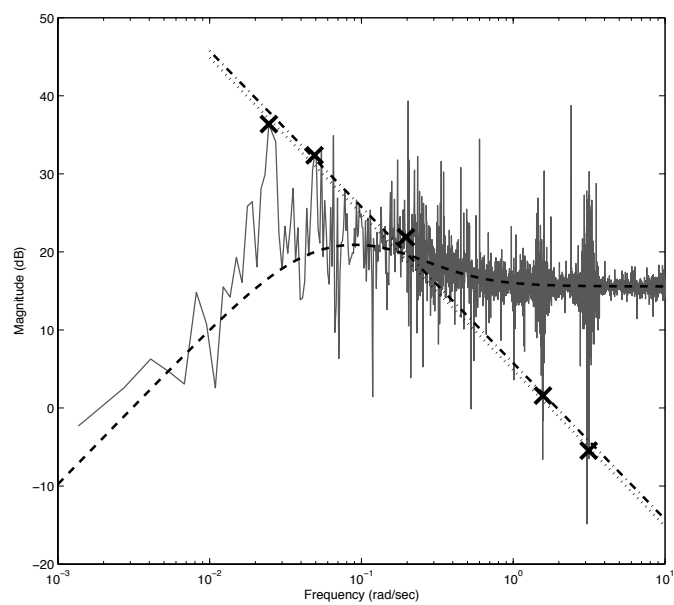

Fig. 6. Results of the experiment in the frequency domain. ETFE (solid line), $-1 / C$ (dashed line), Theoretical model (dotted line), Estimated model (dash-dot line) and $\times$ - location of excitation frequencies.

By utilising only the test frequencies in the identification algorithm it is clearly seen by the dash-dot line in Figure 6 that the system is very similar to that of the theoretical model (dotted line).

Clearly this case study shows that thought must be given to the experiment when a system is operating in closed loop if robust results are to be obtained. It is not sufficient to collect normal operating data and expect to model anything other than the negative inverse of the controller without special care. 


\section{CONCLUSIONS}

This paper has argued that special care is needed to ensure that robust identification is achieved. Special emphasis has been placed on:

- selecting a model class,

- selecting a sampling strategy,

- the effect of sampling on model aliasing,

- limited bandwidth estimation,

- robust experiment design, and

- robust estimation for closed loop data.

\section{Appendix A. PROOF OF THEOREM 6}

Consider any open loop experiment, $\xi_{o l}$. Since the output power is constrained, we have that $\xi_{o l}$ must satisfy

$$
\int \Phi_{y}\left\{\xi_{o l}\right\}=\int\left|G_{o}\right|^{2} \Phi_{u}\left\{\xi_{o l}\right\}+\int\left|H_{o}\right|^{2} \sigma_{w}^{2} \leq K
$$

Next, considering that $\Phi_{u w}\left\{\xi_{o l}\right\}=0$, we have that the covariance matrix for $\hat{\rho}_{N}$ satisfies

$$
P_{\rho}^{-1}\left\{\xi_{o l}\right\}=\int G_{1} G_{1}^{H} \Phi_{u}\left\{\xi_{o l}\right\}
$$

Consider now a closed loop experiment, $\xi_{c l}$, which uses the following control law

$$
u(t)=F_{o}\left(q^{-1}\right) r(t)-C_{o}\left(q^{-1}\right) y(t)
$$

where we take

$$
\Phi_{r}=\mu \Phi_{u}\left\{\xi_{o l}\right\}
$$

and where $\mu$ is a scalar which satisfies

$$
1<\mu \leq \frac{K-\int\left|S_{o} H_{o}\right|^{2} \sigma_{w}^{2}}{K-\int\left|H_{o}\right|^{2} \sigma_{w}^{2}}
$$

Note that it is always possible to find $\mu$ which satisfies equation (A.4) provided there exists a controller, different from zero, such that $\int\left|S_{o} H_{o}\right|^{2}<\int\left|H_{o}\right|^{2}$.

The input and output signals are given by:

$$
\begin{aligned}
& y(t)=G_{o} \bar{S}_{o} S_{o} r(t)+S_{o} H_{o} w(t) \\
& u(t)=\bar{S}_{o} S_{o} r(t)-C_{o} H_{o} S_{o} w(t)
\end{aligned}
$$

where $F_{o}=\bar{S}_{o}\left(q^{-1}\right)$ and $S_{o}\left(q^{-1}\right)=\frac{1}{1+G_{o}\left(q^{-1}\right) C_{o}\left(q^{-1}\right)}$, $\bar{S}_{o}\left(q^{-1}\right)$ is stable and $\bar{S}_{o}\left(q^{-1}\right) S_{o}\left(q^{-1}\right)$ is all pass $\left(\left|\bar{S}_{o} S_{o}\right|^{2}=1\right)$.

This in turn implies that

$$
\begin{aligned}
\Phi_{u}\left\{\xi_{c l}\right\} & =\Phi_{r}+\left|C_{o} S_{o} H_{o}\right|^{2} \sigma_{w}^{2} \\
& =\mu \Phi_{u}\left\{\xi_{o l}\right\}+\left|C_{o} S_{o} H_{o}\right|^{2} \sigma_{w}^{2} \\
& \frac{\left|\Phi_{u w}\left\{\xi_{c l}\right\}\right|^{2}}{\sigma_{w}^{2}}=\left|C_{o} S_{o} H_{o}\right| \sigma_{w}^{2}
\end{aligned}
$$

and

$$
\begin{aligned}
\Phi_{y}\left\{\xi_{c l}\right\} & =\left|\bar{G}_{o}\right|^{2} \Phi_{r}+\left|S_{o} H_{o}\right|^{2} \sigma_{w}^{2} \\
& =\mu\left|\bar{G}_{o}\right|^{2} \Phi_{u}\left\{\xi_{o l}\right\}+\left|S_{o} H_{o}\right|^{2} \sigma_{w}^{2}
\end{aligned}
$$

The output power for the closed loop experiment satisfies

$$
\int \Phi_{y}\left\{\xi_{c l}\right\}=\mu \int\left|\bar{G}_{o}\right|^{2} \Phi_{u}\left\{\xi_{o l}\right\}+\int\left|S_{o} H_{o}\right|^{2} \sigma_{w}^{2}
$$

Hence, considering equations (A.1) and (A.4), we have that

$$
\begin{aligned}
\int \Phi_{y}\left\{\xi_{c l}\right\} & \leq \mu\left(K-\int\left|H_{o}\right|^{2} \sigma_{w}^{2}\right)+\int\left|S_{o} H_{o}\right|^{2} \sigma_{w}^{2} \\
& \leq K
\end{aligned}
$$

which means that the closed loop experiment (A.3), (39) satisfies the output power constraint.

Finally, using part 1 of Lemma 4 and equations (A.5) and (A.6) we have that

$$
\begin{aligned}
P_{\rho}^{-1}\left\{\xi_{c l}\right\} & \geq \int G_{1} G_{1}^{H}\left[\Phi_{u}\left\{\xi_{c l}\right\}-\frac{\left|\Phi_{u w}\left\{\xi_{c l}\right\}\right|^{2}}{\sigma_{w}^{2}}\right] \\
& =\mu \int G_{1} G_{1}^{H} \Phi_{u}\left\{\xi_{o l}\right\}
\end{aligned}
$$

which implies (using equations (A.2) and (A.4)) that:

$$
P_{\rho}^{-1}\left\{\xi_{c l}\right\} \geq \mu P_{\rho}^{-1}\left\{\xi_{o l}\right\}>P_{\rho}^{-1}\left\{\xi_{o l}\right\}
$$

which shows that $\xi_{c l} \stackrel{\rho}{\succ} \xi_{o l}$. This concludes the proof.

\section{REFERENCES}

Agüero, J. C. (2005). System identification methodologies incorporating constraints. $\mathrm{PhD}$ thesis. The University of Newcastle, Australia.

Agüero, J. C. and G. C. Goodwin (2004a). Virtual closed loop identification. Technical report. The University of Newcastle.

Agüero, J. C. and G. C. Goodwin (2004b). Virtual closed loop identification: A Subspace Approach. In: Proceedings of the 43rd IEEE Conference on Decision and Control, CDC. Atlantis, Paradise Island, Bahamas. pp. 364-369.

Agüero, J. C. and G. C. Goodwin (2006). On the optimality of open and closed loop experiments in system identification. In: 45th IEEE Conference on Decision and Control (CDC). San Diego, California.

Agüero, J. C. and G. C. Goodwin (2007). Choosing between open and closed loop experiments in linear system identification. To appear in IEEE Transactions on Automatic Control.

Agüero, J. C., J. I. Yuz and G. C. Goodwin (2007a). Frequency domain identification of mimo state space models using the em algorithm. In: European Control Conference (ECC).

Agüero, J. C., J. Welsh and O. J. Rojas (2007b). On the optimality of binary-open-loop experiments to identify hammerstein systems. In: American Control Conference.

Anderson, B. D. O. and R. L. Kosut (1991). Adaptive robust control: On-line learning. In: Proceedings of the 30th Conference of Decision and Control, $C D C$. 
Åström, K. J., P. Hagander and J. Sternby (1984). Zeros of sampled systems. Automatica 20(1), 3138.

Brillinger, D.R. (1974). Fourier analysis of stationary processes. Proceedings of the IEEE 62(12), 1628-1643.

Brillinger, D.R. (1981). Time Series: Data Analysis and Theory. McGraw-Hill. New York.

Feuer, A. and G.C. Goodwin (1996). Sampling in Digital Signal Processing and Control. Birkhäuser. Boston.

Forssell, U. and L. Ljung (1999). Closed loop identification revisited. Automatica 35, 1215-1241.

Goodwin, G. C. and R. Payne (1977). Dynamic system identification: Experiment design and data analysis. Academic Press.

Goodwin, G. C., J. I. Yuz and M. E. Salgado (2007). Insights into the zero dynamics of sampled-data models for linear and nonlinear stochastic systems. In: European Control Conference ECC.

Goodwin, G. C., S. F. Graebe and M. E. Salgado (2001). Control system design. Prentice Hall. Upper Saddle River, NJ.

Hoyer, D. I. and J. M. Boyes (1990). The highintensity nutating mill- a batch ball milling simulator. Minerals Engineering.

Kiefer, J. (1959). Optimum experiment designs. Journal of the Royal Statistical Society, Series B 21(2), 272-319.

Kosut, R. L. (2000). Iterative adaptive control: Windsurfing with confidence. Model identification and adaptive control. Edited by G. C. Goodwin pp. 201-229.

Lee, W. S., B. D. O. Anderson, I. M. Y. Mareels and R. L. Kosut (1995). On some key issues in the windsurfer approach to adaptive robust control;. Automatica 31(11), 1619-1636.

Lee, W.S., B.D.O. Anderson, R.L. Kosut and I.M.Y Mareels (1993). A new approach to adaptive robust control. International Journal of Adaptive Control and Signal Processing 7(5), 183-211.

Ljung, L. (1993). Some results on identifying linear systems using frequency domain data. In: Proceedings of the 32nd IEEE Conference on Decision and Control. pp. 3534-3538.

Ljung, L. (1999). System identification: Theory for the user. 2nd ed.. Prentice Hall.

McKelvey, T. and L. Ljung (1997). Frequency domain maximum likelihood identification. In: 11th IFAC Symposium on System Identification. Fukuoka, Japan. pp. 1741-1746.

Middleton, R. and G.C. Goodwin (1990). Digital control and estimation: A unified approach. PrenticeHall International.

Napier-Munn, T. J., S. Morell, R. D. Morrison and T. Kojovic (1996). Mineral comminution circuits: Their operation and optimisation. Technical report. Julius Kruttschnitt Mineral Research Centre, University of Queensland.
Pintelon, R. and J. Schoukens (2005). ML identification of closed-loop systems in a specified frequency band. In: Proc. of 16th IFAC World Congress. Prague, Czech Republic.

Pukelsheim, F. (1993). Optimal design of experiments. John Wiley.

Rojas, C. R., G. C. Goodwin, J. S. Welsh and A. Feuer (2007a). Optimal experiment design with diffuse prior information. In: European Control Conference (ECC). Kos, Greece.

Rojas, C. R., J. S. Welsh and G. C. Goodwin (2007b). A receding horizon algorithm to generate binary signals with a prescribed autocovariance. In: American Control Conference.

Rojas, C. R., J. S. Welsh, G. C. Goodwin and A. Feuer (2007c). Robust optimal experiment design for system identification. to appear in Automatica.

Siotani, M. (1967). Some applications of Loewner's ordering on symmetric matrices. Annals of the Institute of Statistical Mathematics 19, 245-259.

Weller, S. R., W. Moran, B. Ninness and A. D. Pollington (2001). Sampling zeros and the EulerFrobenius polynomials. IEEE Transactions on Automatic Control 46(2), 340-343.

Yuz, J. I. (2005). Sampled-Data models for linear and non-linear models. PhD thesis. The University of Newcastle.

Yuz, J. I. and G. C. Goodwin (2006). Sampled-data models for stochastic nonlinear systems. In: 14th IFAC symposium on system identification. Newcastle, Australia.

Yuz, J. I. and G. C. Goodwin (2007). Robust identification of continuous-time systems from sampled data. To appear in "Continuous time system identification from sampled data”. Edited by H. Garnier and L. Wang. Springer-Verlag. 